\title{
The impact of the lung allocation score on short-term transplantation outcomes: A multicenter study
}

Benjamin D. Kozower, MD, ${ }^{a}$ Bryan F. Meyers, MD, ${ }^{b}$ Michael A. Smith, MD, ${ }^{c}$ Nilto C. De Oliveira, MD, ${ }^{d}$ Stephen D. Cassivi, MD, ${ }^{e}$ Tracey J. Guthrie, RN, ${ }^{b}$ Honkung Wang, PhD, ${ }^{f}$ Beverly J. Ryan, ACNP, ${ }^{a}$ K. Robert Shen, MD, Thomas M. Daniel, MD, a and David R. Jones, MDa

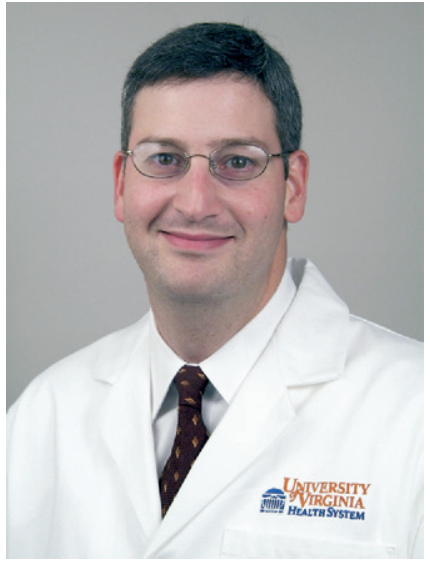

Dr Kozower
From the Department of Surgery, University of Virginia Health System, Charlottesville, $\mathrm{Va}^{\mathrm{a}}$; Department of Surgery, Washington University School of Medicine, St Louis, $\mathrm{Mo}^{\mathrm{b}}$; Department of Cardiothoracic Surgery, University of Southern California, Los Angeles, Calif ${ }^{c}$; Department of Surgery, University of Wisconsin, Madison, Wis ${ }^{\mathrm{d}}$; Department of Surgery, Mayo Clinic, Rochester, Minn ${ }^{\mathrm{e}}$; and Department of Public Health Sciences, University of Virginia Health System, Charlottesville, Va. ${ }^{\mathrm{f}}$

Read at the Eighty-seventh Annual Meeting of The American Association for Thoracic Surgery, Washington, DC, May 5-9, 2007.

Received for publication May 3, 2007; revisions received Aug 13, 2007; accepted for publication Aug 15, 2007.

Address for reprints: Benjamin D. Kozower, MD, University of Virginia Health System, General Thoracic Surgery, PO Box 800679, Charlottesville, VA 22908-0679 (E-mail: bdk8g@virginia.edu).

J Thorac Cardiovasc Surg 2008;135:166-71 $0022-5223 / \$ 34.00$

Copyright (๑) 2008 by The American Association for Thoracic Surgery

doi:10.1016/j.jtcvs.2007.08.044
Objective: The lung allocation score restructured the distribution of scarce donor lungs for transplantation. The algorithm ranks waiting list patients according to medical urgency and expected benefit after transplantation. The purpose of this study was to evaluate the impact of the lung allocation score on short-term outcomes after lung transplantation.

Methods: A multicenter retrospective cohort study was performed with data from 5 academic medical centers. Results of patients undergoing transplantation on the basis of the lung allocation score (May 4, 2005 to May 3, 2006) were compared with those of patients receiving transplants the preceding year before the lung allocation score was implemented (May 4, 2004, to May 3, 2005).

Results: The study reports on 341 patients (170 before the lung allocation score and 171 after). Waiting time decreased from $680.9 \pm 528.3$ days to $445.6 \pm$ 516.9 days $(P<.001)$. Recipient diagnoses changed with an increase in idiopathic pulmonary fibrosis and a decrease in emphysema and cystic fibrosis $(P=.002)$. Postoperatively, primary graft dysfunction increased from $14.1 \%$ $(24 / 170)$ to $22.9 \%(39 / 171)(P=.04)$ and intensive care unit length of stay increased from $5.7 \pm 6.7$ days to $7.8 \pm 9.6$ days $(P=.04)$. Hospital mortality and 1 -year survival were the same between groups $(5.3 \%$ vs $5.3 \%$ and $90 \%$ vs $89 \%$, respectively; $P>.6$ )

Conclusions: This multicenter retrospective review of short-term outcomes supports the fact that the lung allocation score is achieving its objectives. The lung allocation score reduced waiting time and altered the distribution of lung diseases for which transplantation was done on the basis of medical necessity. After transplantation, recipients have significantly higher rates of primary graft dysfunction and intensive care unit lengths of stay. However, hospital mortality and 1-year survival have not been adversely affected.

$\mathrm{T}$ he lung allocation score (LAS) was implemented in May 2005 by the Organ Procurement and Transplantation Network (OPTN). ${ }^{1}$ The LAS dramatically changed lung allocation from a system based purely on waiting time to an algorithm based on survival probability on the waiting list and after transplantation. The impetus for change was the scarcity of suitable donor lungs and the increasing number of deaths for patients on the waiting list. ${ }^{2-4}$

The OPTN began allocating lungs in 1990 on the basis of blood type and the amount of time candidates had spent on the waiting list. ${ }^{5}$ In 1995, a minor change was made to this system when 90 days of waiting time were added for patients with idiopathic pulmonary fibrosis to offset the increased risk of mortality on the waiting list. In 1998, the Department of Health and Human Services published the Final Rule. ${ }^{6}$ This required the OPTN to emphasize the broader sharing of organs, reduce 


\section{Abbreviations and Acronyms \\ ICU = intensive care unit \\ LAS = lung allocation score \\ OPTN $=$ Organ Procurement and Transplantation Network}

the use of waiting time as an allocation criterion, and create an allocation system based on objective medical criteria and measures of medical urgency.

The LAS was developed by multivariate modeling and was approved by the OPTN in 2004. The three main objectives were as follows: (1) reduce the number of deaths on the lung transplant waiting list, (2) increase transplant benefit for lung recipients, and (3) ensure the efficient and equitable allocation of lungs to active transplant candidates. $^{7}$ The LAS assigns a score to all candidates over the age of 12 years ranging from 0 to 100 . It is a weighted combination of predicted risk of death during the following year on the waiting list and the predicted likelihood of survival during the first year after transplantation.

The purpose of this study was to evaluate the impact of the LAS system on the waiting list and short-term outcomes after lung transplantation. Many lung transplant surgeons have the impression that the LAS has increased the complexity of the cases and their complication rates. Our hypothesis was that the LAS would decrease waiting time for recipients but would also increase morbidity and mortality after transplantation.

\section{Patients and Methods \\ Transplant Recipients}

A multicenter retrospective cohort study was performed with data from five academic medical centers: University of Virginia, Washington University, University of Southern California, University of Wisconsin, and the Mayo Clinic. The two cohorts were patients undergoing transplantation the year preceding the introduction of the LAS (May 4, 2004, to May 3, 2005) (pre-LAS group) and patients undergoing transplantation the year after the LAS was implemented (May 4, 2005, to May 3, 2006) (LAS group). In an effort to compare the severity of illness between the groups, the LAS was calculated for the pre-LAS group using the appropriate variables available close to the time of transplant. Primary graft dysfunction was defined according to the International Society of Heart and Lung Transplantation definition: arterial oxygen tension/ inspired oxygen fraction less than 300 and a chest radiograph with a characteristic diffuse bilateral infiltrate. ${ }^{8}$ The human studies committees at each institution granted approval for this research.

\section{Waiting List Estimates}

Data from the waiting lists at the five institutions were gathered for the same two groups. To calculate the percentage of patients dying on the waiting list, we estimated the size of the waiting list for both groups. Because the waiting lists are dynamic, this is not a straightforward process. We identified the actual number of patients on the waiting lists at 4 interval time points for both cohorts (May 15, August 15, November 15, and February 15). These numbers were averaged (total number divided by 4 ) to estimate the size (denominator) of the waiting list. The numerator was the actual number of patients who died on the waiting lists.

\section{Statistical Analysis}

Categorical variables were compared by the $\chi^{2}$ test. Continuous variables were compared with the Student $t$ test or Kruskal-Wallis test where appropriate. Estimates of the cumulative death rate at 1 year were calculated by the Kaplan-Meier method, and the survival differences between the pre-LAS and LAS groups were assessed by the log-rank test. Short-term results were controlled for diagnosis by the Mantel-Haenszel test. All data analysis was performed with SAS 9.1.3 software (SAS Institute, Inc, Cary, NC).

\section{Results}

\section{Patient Characteristics}

There were 170 patients in the pre-LAS group and 171 in the LAS group. The numbers of patients per group from each institution were as follows: Washington University 59 and 52, University of Southern California 37 and 38, University of Virginia 31 and 35, University of Wisconsin 34 and 33, and Mayo Clinic 9 and 13. The baseline characteristics are listed in Table 1. The pre-LAS group had fewer retransplants and a lower calculated LAS $(P<.05)$. The recipient diagnoses changed significantly with the initiation of the LAS (Table 2). There were an increase in the number of patients with idiopathic pulmonary fibrosis and a decrease in patients with chronic obstructive pulmonary disease and cystic fibrosis $(P=.002)$.

\section{TABLE 1. Baseline characteristics}

\begin{tabular}{lccc}
\hline & Pre-LAS $(\mathbf{n}=\mathbf{1 7 0})$ & LAS (n= 171) & $\boldsymbol{P}$ value \\
\hline Age (y) & $49.2 \pm 14.0$ & $51.2 \pm 13.0$ & .16 \\
Gender (female) & $80(47.1 \%)$ & $80(46.8 \%)$ & .96 \\
Procedure (bilateral lung transplant) & $111(65.3 \%)$ & $106(62.0 \%)$ & .2 \\
Retransplantation & $3(1.8 \%)$ & $10(5.8 \%)$ & .05 \\
Ischemic time (first lung in minutes) & $285.2 \pm 97.9$ & $271.0 \pm 99.4$ & .20 \\
LAS & $35.4 \pm 8.2$ & $42.5 \pm 15.2$ & $<.001$ \\
\hline
\end{tabular}

LAS, Lung allocation score. 
TABLE 2. Recipient diagnosis

\begin{tabular}{lcc}
\hline & Pre-LAS $(\mathbf{n}=\mathbf{1 7 0})$ & LAS $(\mathbf{n}=\mathbf{1 7 1})$ \\
\hline Etiology of end-stage lung disease & & \\
COPD & $78(45.9 \%)$ & $58(33.9 \%)$ \\
Cystic fibrosis & $39(22.9 \%)$ & $22(12.9 \%)$ \\
Idiopathic pulmonary fibrosis & $25(14.7 \%)$ & $42(24.6 \%)$ \\
Pulmonary hypertension & $7(4.1 \%)$ & $4(2.3 \%)$ \\
Other & $21(12.4 \%)$ & $45(26.3 \%)$ \\
\hline
\end{tabular}

$L A S$, Lung allocation score; COPD, chronic obstructive pulmonary disease.

\section{Waiting List}

Implementation of the LAS reduced the waiting time for transplant recipients $(680.9 \pm 528.3$ days for pre-LAS vs $445.6 \pm 516.9$ days for LAS; $P<.001$ ). The percentage of patients dying on the waiting list decreased from $15.3 \%$ (74/485) for the pre-LAS group to $11.3 \%(51 / 450)$ for the LAS group $(P=.08)$. However, the absolute number of patients dying on the waiting list decreased from 74 to 51 , a $30 \%$ decrease.

\section{Surgical Morbidity}

Surgical morbidity is illustrated in Table 3 . Primary graft dysfunction was higher in the LAS group $(P<.04)$. The total number of days mechanically ventilated and lengths of stay in the intensive care unit (ICU) were also higher in the LAS group $(3.3 \pm 5.1$ days vs $6.8 \pm 14.4$ days; $P=.004$; and $5.7 \pm 6.7$ days vs $7.8 \pm 9.3$ days; $P=.02$, respectively). However, the total number of hospital days was similar between the two groups $(22.8 \pm 34.0$ days vs 22.2 \pm 22.5 days; $P=.86$ ).

To determine whether elevated pulmonary artery pressure was associated with primary graft dysfunction, we compared the mean and systolic pulmonary artery pressures between the groups and between those patients with and without graft dysfunction. The mean and systolic pulmonary artery pressures were similar between the pre-LAS and LAS groups (27.5 vs $28.9 \mathrm{~mm} \mathrm{Hg}$ and 40.1 vs $43.7 \mathrm{~mm} \mathrm{Hg}$, respectively $(P>.19)$. In addition, there was a trend but no significant relationship between systolic pulmonary artery pressure and the presence of graft failure in this study ( 41.2 vs $50.5 \mathrm{~mm} \mathrm{Hg} ; P=.14)$.
When the groups were adjusted for diagnosis, primary graft dysfunction and days mechanically ventilated were no longer associated with group $(P>.2)$. When the rates of primary graft dysfunction for patients with pulmonary fibrosis were examined, there was no significant difference between the groups $(32 \%[8 / 25]$ vs $22 \%$ [9/41]; $P=.4)$. However, ICU length of stay continued to be associated with group $(P=.04)$.

\section{Mortality}

Perioperative mortality was defined as a death within 30 days of transplantation or during the same hospitalization. Perioperative mortality was not different between the two groups $(5.3 \%[9 / 170]$ vs $5.3 \%[9 / 171] ; P=.99)$. The Kaplan-Meier 1-year survival was also similar between the two groups $(86.4 \%$ vs $89.9 \% ; P=.6)$.

\section{Conclusions}

The LAS significantly changed the allocation process for donor lungs in the United States. It was initiated because the previous system, based on waiting time, failed to accommodate patients with a rapidly deteriorating course who could not tolerate the prolonged waiting times and who were more likely to die on the waiting list. ${ }^{2,9,10}$ The LAS algorithm attempts to balance waiting list urgency with posttransplant survival. Because these vary among patients with different lung diseases, diagnosis is included as a variable in the LAS calculation. This report demonstrates a marked shift in the lung diseases being treated by transplantation with an increase in idiopathic pulmonary fibrosis and

TABLE 3. Perioperative morbidity

\begin{tabular}{lccc}
\hline & Pre-LAS $(\mathbf{n}=\mathbf{1 7 0})$ & LAS (n= 171) & $\boldsymbol{P}$ value \\
\hline Primary graft dysfunction & $24(14.1 \%)$ & $39(22.9 \%)$ & .04 \\
ECMO & $8(4.7 \%)$ & $7(4.1 \%)$ & .78 \\
Reoperation & $19(11.2 \%)$ & $25(14.3 \%)$ & .32 \\
Airway complications & $9(6.4 \%)$ & $10(7.8 \%)$ & .67 \\
Dialysis & $10(6.0 \%)$ & $11(6.4 \%)$ & .84 \\
Reintubation & $32(19.3 \%)$ & $26(15.9 \%)$ & .41 \\
\hline
\end{tabular}

LAS, Lung allocation score; ECMO, extracorporeal membrane oxygenation. 
a decrease in cystic fibrosis and emphysema. These changes are consistent with the goals of the LAS inasmuch as patients with idiopathic pulmonary fibrosis had a higher mortality on the waiting list under the previous allocation system. ${ }^{4,10}$ However, this contradicts an earlier report by Lingaraju and colleagues, ${ }^{11}$ which simulated the effects of the LAS on the lung diseases being treated by transplantation. Their simulation showed that patients with pulmonary fibrosis had improved rankings and patients with emphysema had worse rankings but the number of transplants for each disease category did not change significantly.

Importantly, our results demonstrate that the LAS reduced the waiting time for transplantation by $35 \%(P<$ .001). It will be very interesting to see whether this is sustainable as data from the LAS system mature. One could hypothesize that if the number of donor lungs remains fixed, sicker patients will populate the waiting list and waiting times may begin to increase over time. Our data would not reflect this inasmuch as there was a significant increase in the number of patients with pulmonary fibrosis receiving transplants during the first year of the LAS system.

Our estimation of the percent of patients dying on the waiting list showed that the LAS group may have fewer deaths $(15.3 \%$ [74/485] pre-LAS group vs $11.3 \%$ [51/450] LAS group; $P=.08$ ). The true number is dynamic and very difficult to identify precisely. If one looks only at the number of deaths on the waiting list, it is not easy to compare groups because the denominator, the total number of patients on the waiting list, is unknown. However, the absolute number of patients dying on the waiting list did decrease from 74 to 51 , a $30 \%$ decrease.

In addition, there are patients listed now who would never have been listed before because they had little chance of surviving on the waiting list in the old system. These "sick" patients might die on the waiting list in the new LAS system, but they would never have been listed in the old system. In this way, the LAS system might inflate death rates. On the other hand, the old system denominator was inflated with relatively healthier patients with emphysema who had little chance of undergoing transplantation in the current LAS system. It is quite likely that these patients are not even listed now because they would have a low LAS and placing them on the list to accrue time is no longer advantageous. Thus, the change in systems not only changes who dies on the waiting list, but it also changes who is listed in the first place, thus making a difference in mortality very difficult to interpret.

The pre-LAS and LAS groups were comparable with respect to age, gender, procedure performed, and ischemic time (Table 1). However, the score of the LAS group had more retransplants and a higher LAS than had the calculated score for the pre-LAS group. This is an expected difference because the donor lungs in the LAS group were allocated to patients with higher LASs. The International Society of
Heart and Lung Transplantation, along with other reported series, have identified retransplantation as a significant risk factor for primary graft dysfunction. ${ }^{12,13}$ In addition, diagnoses of pulmonary fibrosis and pulmonary hypertension are established risk factors for the development of primary graft dysfunction. Our data demonstrate that as the number of transplants for pulmonary fibrosis increased, so did the rates of primary graft dysfunction. However, when we controlled for diagnoses, the rates of primary graft dysfunction were no longer different between the groups. Therefore, much of the increased morbidity seen in the LAS group is due to the shift in lung diseases being treated by transplantation.

The increase in primary graft dysfunction with the LAS group explains the doubling of the length of mechanical ventilation and the increase in ICU length of stay. Interestingly, although primary graft dysfunction is the most important predictor of postoperative mortality and was present in almost $25 \%$ of the LAS group, there was no difference in mortality between the cohorts in the study. ${ }^{14}$ In fact, the mortality for both groups was only $5.3 \%(P=.99)$. It is also important to note that there was no significant relationship between elevated pulmonary artery pressure and primary graft dysfunction in this study.

\section{Limitations of the Study}

Limitations of this study are that it is a retrospective review with a short follow-up. The real impact of the LAS will not be realized for several years. It is important to understand that the LAS system is dynamic. It was designed to update the models every 6 months to include the most recent data with at least 3 years of follow-up. ${ }^{5}$

In conclusion, this multicenter retrospective study of short-term outcomes supports that the LAS is achieving its objectives. The LAS has reduced waiting time and altered the lung diseases being treated by transplantation. Although transplant recipients have higher rates of primary graft dysfunction and longer stays in the ICU, the overall hospital stay and mortality are not affected. However, we do not know whether the LAS will reduce mortality on the waiting list and what the long-term effects of these changes will be. We do know that if the LAS system increases the rates of primary graft dysfunction and the length of ICU stay, it will increase the medical and financial resources required to care for these patients. Finally, despite increased morbidity, the 1-year survival approached $90 \%$ in the LAS group. This is an excellent early result for a sick group of patients, but longer follow-up is needed to draw definitive conclusions about the success of the LAS system and to modify the algorithm with more comprehensive data.

\section{References}

1. Levine GN, McCullough KP, Rodgers AM, Dickinson DM, Ashby VB, Schaubel DE. Analytical methods and database design: impli- 
cations for transplant researchers, 2005. Am J Transplant. 2006;6: 1228-42.

2. Egan TM, Kotloff RM. Pro/con debate: lung allocation should be based on medical urgency and transplant survival and not on waiting time. Chest. 2005;128:407-15.

3. De Meester J, Smits JM, Persijn GG, Haverich A. Listing for lung transplantation: life expectancy and transplant effect, stratified by type of end-stage lung disease, the Eurotransplant experience. J Heart Lung Transplant. 2001;20:518-24

4. Travaline JM, Cordova FC, Furukawa S, Criner GJ. Discrepancy between severity of lung impairment and seniority on the lung transplantation list. Transplant Proc. 2004;36:3156-60.

5. Egan TM, Murray S, Bustami RT, Shearon TH, McCullough KP, Edwards LB, et al. Development of the new lung allocation system in the United States. Am J Transplant. 2006;6:1212-27.

6. Department of Health and Human Services. Organ procurement and transplantation network, HHS: final rule; 42 CFR-part 121. Fed Regist. 1999:56650-61.

7. D'Alonzo GE, Barst RJ, Ayres SM, Bergofsky EH, Brundage BH, Detre KM, et al. Survival in patients with primary pulmonary hypertension. results from a national prospective registry. Ann Intern Med. 1991;115:343-9.

8. Christie JD, Carby M, Bag R, Corris P, Hertz M, Weill D, ISHLT Working Group on Primary Lung Graft Dysfunction. Report of the ISHLT Working Group on Primary Lung Graft Dysfunction part II: Definition. A consensus statement of the International Society for Heart and Lung Transplantation. J Heart Lung Transplant. 2005;24: $1454-9$.

9. De Meester J, Smits JM, Persijn GG, Haverich A. Lung transplant waiting list: differential outcome of type of end-stage lung disease, one year after registration. J Heart Lung Transplant. 1999;18:563-71.

10. Pierson RN, Milstone AP, Loyd JE, Lewis BH, Pinson CW, Ely EW. Lung allocation in the United States, 1995-1997: an analysis of equity and utility. J Heart Lung Transplant. 2000;19:846-51.

11. Lingaraju R, Blumenthal NP, Kotloff RM, Christie J, Ahya VN, Sager JS, et al. Effects of lung allocation score on waiting list rankings and transplant procedures. J Heart Lung Transplant. 2006;25:1167-70.

12. Kozower BD, Sweet SC, de la Morena M, Schuler P, Guthrie TJ, Patterson GA, et al. Living donor lobar grafts improve pediatric lung retransplantation survival. J Thorac Cardiovasc Surg. 2006;131: $1142-7$.

13. Barr ML, Kawut SM, Whelan TP, Girgis R, Böttcher H, Sonett J, et al. Report of the ISHLT Working Group on Primary Lung Graft Dysfunction part IV: recipient-related risk factors and markers. J Heart Lung Transplant. 2005;24:1468-82.

14. Arcasoy SM, Fisher A, Hachem RR, Scavuzzo M, Ware LB, ISHLT Working Group on Primary Lung Graft Dysfunction. Report of the ISHLT Working Group on Primary Lung Graft Dysfunction part V: predictors and outcomes. J Heart Lung Transplant. 2005;24:1483-8.

\section{Discussion}

Dr Kenneth R. McCurry (Pittsburgh, Pa). I congratulate Dr Kozower and his colleagues on their work. I think the impact of the LAS on posttransplant outcomes as well as waiting list mortality are critical issues that deserve a great deal of attention. This paper is the first to address both sides of this equation in a comprehensive fashion.

Dr Kozower and his colleagues have presented data from their five institutions demonstrating a significant change in the distribution of lung transplants by recipient diagnosis since implementation of the LAS system, with an increase in the percentage of transplants being performed for idiopathic pulmonary fibrosis from $15 \%$ to $25 \%$ and a concomitant decrease in the percentage of transplants being performed for chronic obstructive pulmonary disease from $46 \%$ to $34 \%$. As Dr Kozower has stated, these data are certainly anticipated and indeed the intended effect of the LAS system. They are also consistent with, although somewhat less dramatic than, the initial 6-month analysis of United Network for Organ Sharing data in October of 2005, where about $46 \%$ of the transplants performed in the United States during the first 6 months after initiation of the LAS were performed for restrictive lung disease. At our own center in Pittsburgh, approximately $35 \%$ of our patients received transplants for idiopathic pulmonary fibrosis compared with about $18 \%$ previously. This distribution will obviously vary a little bit depending on the local referral practices and the associated medical programs.

Also, interestingly, 2 weeks ago at the annual meeting of the International Society for Heart and Lung Transplantation, the Johns Hopkins Lung Transplant Program presented an abstract evaluating global US 1-year survival under the new LAS system. In their published abstract, they reported on 1486 lung transplants that were performed in the United States from May 4, 2005, which was the start date of the LAS system, through July 15, 2006. In the group of patients with an LAS less than 44, they found a 1-year survival of $73 \%$, whereas the group with an LAS greater than or equal to 44.4 had a significantly lower 1-year survival of approximately $56 \%$. In the five academic medical centers represented in Dr Kozower's paper, the mean LAS in the LAS era was $42 \pm 15.2$, yet they had a 1-year survival of $86.4 \%$. These are certainly excellent outcomes and the centers represented should be congratulated in that regard. This discrepancy does, however, raise the question of whether these excellent outcomes can be universally achieved in the undoubtedly sicker patient population that is and will receive transplantation under the LAS system now and in the future. As Dr Kozower has noted, however, the design of the LAS system was not only to consider the medical urgency of potential recipients, that is, those most likely to die without a transplant, but also to consider net transplant benefit, taking into consideration posttransplant survival as well as waiting list mortality. Thus, it is imperative to evaluate the effect of the LAS system on waiting list mortality, as you have done.

Intuitively, and certainly from personal experience, I do believe that the waiting list mortality has declined under the LAS system. Indeed, I believe that in 2005, when the LAS system was implemented in the United States, there were about 350 deaths on the waiting list that year compared with about 500 per year for the previous 4 to 5 years. I do, however, have a question regarding the methodology that you used to calculate waiting list mortality in your paper. You describe estimating the denominator by determining the number of patients on the waiting list at 4 different time points and then averaging these numbers to determine the average number of patients waiting over the year, while the numerator was the actual numbers of deaths while waiting. This method does not take into account those patients who were removed from the waiting list owing to progression of disease to a point of not being transplantable. This event would also be a failure, I would think, of whatever allocation system was in place at the time. Would it not have been simpler and indeed more thorough to determine the fate of each patient who was on the waiting list over the study period? In this fashion, there would be four possible outcomes for each patient - transplantation, death waiting, removed from the list owing to progression of disease, or continued waiting on the list-to allow a more clear understanding of what happens to patients on the lung transplantation waiting list. 
Dr Kozower. Thank you for your comments and for an excellent first question. I agree with you that it would definitely be more thorough to evaluate the outcome for every patient who was listed for transplant. However, I am not sure it would be simpler. With this retrospective cohort design, it was difficult for us to determine how many patients were on the list at specific time points, and some centers do not have records of patients listed for transplantation. Given these limitations, our method of determining how many patients were on the waiting lists seems reasonable.

Dr McCurry. I think it is going to be a critical issue going forward.

My second question is, in your presentation you reported that the rate of primary graft dysfunction as well as days of mechanical ventilation and days in the ICU were significantly greater in the LAS era than in the pre-LAS era, although only ICU length of stay remained significant after adjustment for diagnosis. Can you expand on the grade of primary graft dysfunction that you included in your paper? As you know, the International Society for Heart ad Lung Transplantation now has 4 different grades, 0 through 3, for primary graft dysfunction. Also, can you expand on why you think this rate was increased in the LAS era? Did you look at other potential recipient confounding factors, such as presence of pulmonary hypertension, use of cardiopulmonary bypass, or transfusion requirements? Did you look at donor demographics to assure that they were equal between the two groups?

Dr Kozower. We defined primary graft dysfunction as grades 2 and 3 , that is, an arterial oxygen tension/inspired oxygen fraction less than 300 and a chest radiograph with the diffuse infiltrates. This occurred within the first 3 days. As to why patients in the LAS era had more primary graft dysfunction, I think for our series much of that was shown to be due to the increase in patients requiring transplantation for idiopathic pulmonary fibrosis.
The third part of your question is a good one. We did not look at pulmonary artery pressures in both groups, but that is included in the LAS score, which was higher in the LAS group. We did look at the use of cardiopulmonary bypass and it was quite similar between the two groups. In the pre-LAS era it was 34\%, and for the LAS group it was $37 \%$, with a $P$ value of .8 .

Dr McCurry. Finally, what is your clinical impression as to why ICU stay was prolonged? Were there more infectious complications in the sicker patient population in the LAS, or did you have more patients who were in the hospital awaiting transplantation preoperatively? What was your clinical impression as to why they stayed in the ICU longer?

Dr Kozower. My clinical impression is that these patients are definitely sicker preoperatively. We have shown that there was a significant increase in the LAS between the groups. You raise an excellent point about infectious complications and in-house patients, but we did not collect those data.

Dr Thomas M. Egan (Chapel Hill, NC). I congratulate you and your other institutions for outstanding results in sicker patients.

I want to echo Dr McCurry's comment about pulmonary hypertension. Presumably, you did not change your donor acceptance criteria from 1 year to the next, and so the incidence of primary graft failure has to be recipient factors and not donor factors. That would imply that you have to look at what the donor factors were. Although you did not show a difference in diagnosis, my bet would be that there is a difference in pulmonary pressures in those patients, and pulmonary hypertension is a risk factor for primary graft dysfunction.

Dr Kozower. I agree with you, Dr Egan. We did not examine pulmonary artery pressure independently, but it is included in the LAS algorithm, which was increased in the LAS group. 